\title{
Geschäftsbericht 2017 der FMH Services Genossenschaft
}

2017 haben wir intensiv daran gearbeitet, unsere bestehenden Angebote weiter zu verfeinern und auszubauen. Mit verstärkter juristischer Beratung, die auf eigenen Spezialisten sowie ergänzend auf einem schweizweiten Netzwerk mit auf die Ärzteschaft spezialisierten Juristen basiert, decken wir ein aktuelles Bedürfnis ab. Die bereits in der Startphase erreichte hohe Zahl der durchgeführten Rechtsberatungen ist erfreulich. Intensiviert haben wir auch unsere Präsenz für Beratungen in der Westschweiz: Seit anfangs 2018 ist FMH Services (Consulting) in Lausanne mit einer eigenen Vertretung präsent und damit auch räumlich näher bei unseren französischsprachigen Kundinnen und Kunden.

Das Geschäftsergebnis 2017 der FMH Services Genossenschaft ist geprägt durch eine erfreuliche Entwicklung der Erträge bei den Finanzanlagen. Auch dank budget-

Dr. med. Beat Bumbacher, MLaw Präsident der Verwaltung FMH Services Genossenschaft

Dr méd. Beat Bumbacher, MLaw Président du conseil d'administration de la coopérative FMH Services konformen Aufwänden ist das Gesamtergebnis sehr positiv. Die Bilanz der FMH Services Genossenschaft weist weiterhin eine solide Struktur aus, welche bei guter Gelegenheit auch weitere Möglichkeiten zum Ausbau der Geschäftstätigkeit zum Nutzen unserer Genossenschafterinnen und Genossenschafter zulässt. Der Geschäftsbericht 2017 kann auf unserer Website (www. fmhservices.ch) abgerufen werden.

Ich danke allen Beteiligten in unserer Organisation, darin eingeschlossen sämtliche Mitglieder unseres engmaschigen Partnernetzes, für ihre erfolgreiche Tätigkeit im vergangenen Jahr.

Ihnen, liebe Genossenschafterinnen und Genossenschafter, danke ich für Ihre Treue und Kundschaft. Wir werden weiter daran arbeiten, Sie bei der nachhaltigen und erfolgreichen Planung und Gestaltung Ihrer $\mathrm{Zu}$ kunft zu unterstützen.

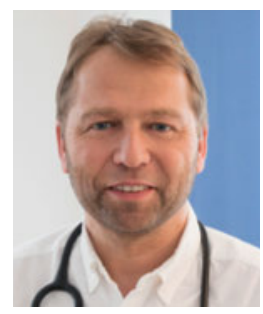

\section{Rapport de gestion 2017 de la coopérative FMH Services}

En 2017, nous avons intensifié nos efforts pour affiner et élargir nos prestations: avec un service de conseil juridique renforcé, qui s'appuie sur nos propres spécialistes et un réseau suisse de juristes spécialisés dans le domaine médical, nous couvrons les besoins actuels. Le nombre élevé de conseils effectués dans la phase de démarrage est réjouissant. Nos efforts se sont aussi concentrés sur notre présence en Suisse romande: depuis début 2018, FMH Services (Consulting) s'est installée à Lausanne, ce qui assure une plus grande proximité géographique avec nos clients francophones.

Le résultat d'exploitation 2017 de la coopérative FMH Services est marqué par une évolution réjouissante des produits des placements financiers. Avec une évolution des dépenses conforme au budget, le résultat global de la coopérative est très positif. Le bilan de la coopérative FMH Services reste solide, ce qui permettra, le cas échéant, d'élargir l'activité commerciale à l'avantage de nos sociétaires. Notre rapport de gestion 2017 peut être téléchargé sur notre site internet (www. fmhservices.ch).

Je tiens à remercier ici toutes les parties prenantes dans notre organisation, ainsi que tous les membres de notre réseau de partenaires pour leur engagement tout au long de l'année.

Chers Sociétaires, je vous remercie de votre fidélité et de votre confiance. Nous continuerons d'œuvrer pour que notre offre de prestations puisse aussi à l'avenir satisfaire à vos attentes. 